\title{
MARINE OMEGA-3 FATTY ACIDS IN THE PREVENTION \\ OF CARDIOVASCULAR DISEASE
}

\author{
Trevor A Mori \\ School of Medicine, Royal Perth Hospital Unit, \\ University of Western Australia and the Cardiovascular Research Centre, \\ Perth, Western Australia, Australia.
}

$\underline{\text { Address correspondence: }}$

Professor Trevor A Mori, School of Medicine, Medical Research Foundation Building, Box X 2213 GPO, Perth, Western Australia 6847.

Telephone: 61892240273

Fax: 61892240246

Email: trevor.mori@uwa.edu.au

Keywords: omega-3 fatty acids, fish oils, cardiovascular disease, eicosanoids 


\section{ABSTRACT}

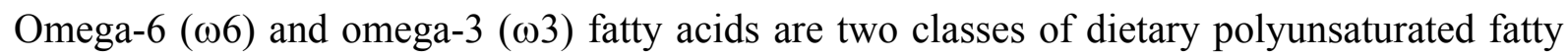

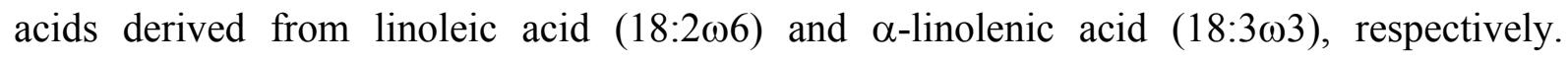
Enzymatic metabolism of linoleic and $\alpha$-linolenic acids generates arachidonic acid $(20: 4 \omega 6)$ and eicosapentaenoic acid (20:5 $\omega 3$; EPA), respectively, both of which are substrates for enzymes that yield eicosanoids with multiple and varying physiological functions. Further elongation and desaturation of EPA yields the 22-carbon fatty acid docosahexaenoic acid

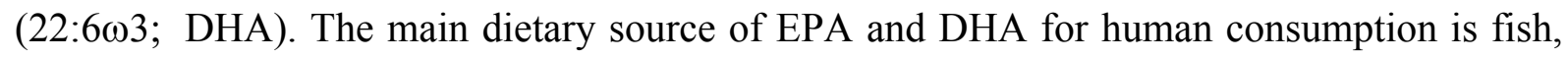
especially oily fish.

There is considerable evidence that EPA and DHA are protective against cardiovascular disease (heart disease and stroke), particularly in individuals with pre-existing disease. $\omega 3$ Fatty acids benefit multiple risk factors including blood pressure, blood vessel function, heart function and blood lipids, and they have antithrombotic, anti-inflammatory and antioxidative actions. $\omega 3$ Fatty acids do not adversely interact with medications. Supplementation with $\omega 3$ fatty acids is recommended in individuals with elevated blood triglyceride levels and patients with coronary heart disease. A practical recommendation for the general population is to increase $\omega 3$ fatty acid intake by incorporating fish as part of a healthy diet that includes increased fruits and vegetables, and moderation of salt intake. Health authorities recommend the general population should consume at least two oily fish meals per week. 


\section{Introduction}

Observational studies from more than forty years ago showed the Greenland Inuit population had a low incidence of coronary artery disease and a reduced prevalence of arthritis, psoriasis, asthma and diabetes, that most likely related to their lifestyle and in particular to their distinctive diet [1-3]. The Greenland Inuit diet derived mainly from cold water fish and artic mammals such as seal and whale. It contained higher protein and fat, lower carbohydrates and significantly greater amounts of omega-3 ( $\omega 3)$ polyunsaturated fats, particularly

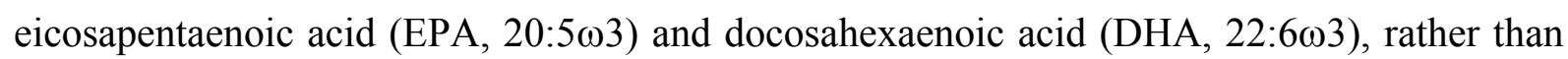

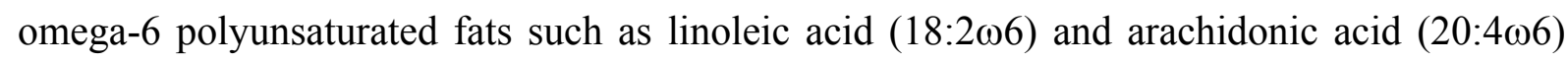
that are characteristic of the Western diet [2]. The plasma fatty acid profile of the Inuit population was consistent with these differences in dietary fats. They had a more favourable lipid profile, typically lower in triglycerides, cholesterol, low density lipoprotein cholesterol (LDL-C) and very low density lipoprotein cholesterol (VLDL-C), and higher in high density lipoprotein cholesterol (HDL-C). They also had reduced blood clotting and thrombosis [1-3].

\section{Sources, Biochemistry and Metabolism Of $\omega 3$ and $\omega 6$ Fatty Acids}

$\omega 6$ and $\omega 3$ Fatty acids represent two classes of polyunsaturated fatty acids that derive from

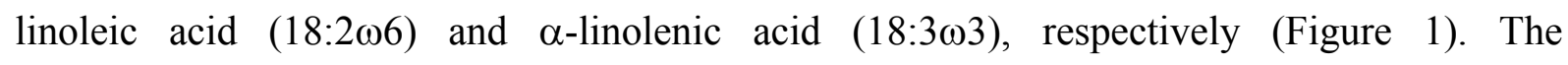
nomenclature for the $\omega 6$ and $\omega 3$ fatty acids relates to the presence of a double bond at the sixth or third carbon, respectively, from the methyl terminus of the fatty acid chain. Metabolism of linoleic and $\alpha$-linolenic acids by desaturase and elongase enzymes generates arachidonic acid and EPA, respectively. Both arachidonic acid and EPA are substrates for cyclooxygenase, lipoxygenase and cytochrome P450 enzymes, yielding eicosanoids and mediators of inflammation resolution with multiple and varying physiological functions. Further metabolism of EPA yields the 22-carbon fatty acid DHA. There are numerous reports 
that the eicosanoids derived from EPA are generally less biologically active than those from arachidonic acid [4], or they antagonise the action of those metabolites derived from arachidonic acid [5].
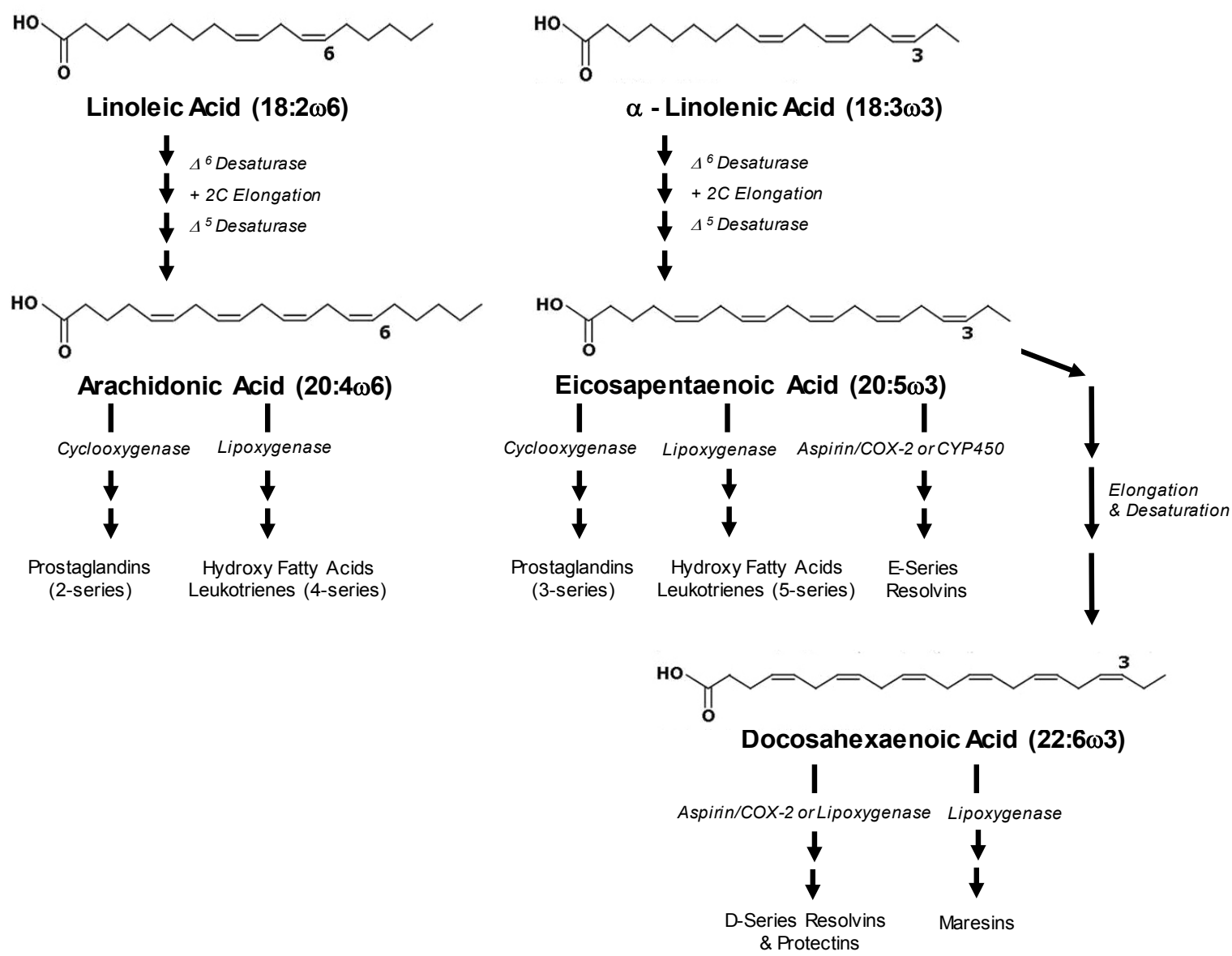

FIGURE 1: Metabolism of linoleic acid and a-linolenic acid to arachidonic acid and eicosapentaenoic acid, respectively. Both arachidonic acid and eicosapentaenoic acid are substrates for cyclooxygenase, lipoxygenase and cytochrome P450 enzymes, yielding eicosanoids and lipid mediators of inflammation resolution. Eicosapentaenoic acid is further elaborated to docosahexaenoic acid.

Western diets are generally more abundant in $\omega 6$ fatty acids that are found mainly in vegetable oils rich in linoleic acid. The dietary intake of $\alpha$-linolenic acid, found in plant oils 
such as linseed oil $(\sim 53 \%)$, canola oil $(\sim 10 \%)$, soybean oil $(\sim 7 \%)$ and walnut oil $(\sim 10 \%)$, is relatively low. Humans are unable to pre-form linoleic acid and $\alpha$-linolenic acid, and thus these fatty acids are termed "essential" dietary fatty acids. Humans are also unable to convert $\omega 6$ fatty acids to $\omega 3$ fatty acids and have a very limited capacity to convert $\alpha$-linolenic acid to EPA and DHA [6]. Thus, the main dietary sources of EPA and DHA are fish especially oily fish such as mackerel, salmon, cod, mullet, herring and flounder.

\section{Evidence from Population Studies}

A number of population studies and meta-analyses have examined the relationship between $\omega 3$ fatty acids and cardiovascular disease [7-15]. In a meta-analysis comprising 15,806 patients, Bucher et al [7] showed that $\omega 3$ fatty acids associated with a $30 \%$ reduction in fatal myocardial infarction and sudden death $(\mathrm{P}<0.01)$, and a $20 \%$ reduction in overall mortality $(\mathrm{P}<0.001)$. Whelton et al [10] in 19 studies comprising 228,864 participants, showed that fish consumption associated with a risk reduction of $17 \%$ for fatal coronary heart disease (CHD) $(\mathrm{P}<0.005)$ and $14 \%$ for total CHD $(\mathrm{P}<0.005)$. A meta-analysis by He et al [8] that included 222,364 individuals, showed the relative risk for CHD mortality was reduced by $23 \%$ in those that ate fish 2-4 times/week, compared with individuals who either never ate or consumed less than one fish meal per month.

Two recent meta-analyses by Maki et al [15] and Alexander et al [14] assessing use of EPA and/or DHA supplements provide additional support that these fatty acids confer benefits on cardiovascular health. In an analysis of 14 randomised controlled trials including 71,899 subjects, Maki et al [15] reported an $8.0 \%(\mathrm{P}=0.015)$ lower risk for cardiac death with

$\omega 3$ fatty acids versus controls. A meta-analysis by Alexander et al [14] that included 8 randomised controlled trials and 16 prospective cohort studies incorporating 732,000 individuals examined EPA+DHA from foods or supplements and CHD, including myocardial 
infarction, sudden cardiac death, coronary death, and angina. The data showed that among randomised controlled trials there was a non-significant $6 \%$ reduction in CHD risk with EPA+DHA. However, subgroup analyses indicated a statistically significant CHD risk reduction with EPA+DHA among higher-risk populations, including participants with elevated triglyceride levels (16\% reduction) and elevated low-density lipoprotein cholesterol (14\% reduction). Meta-analysis of data from prospective cohort studies showed a statistically significant $18 \%$ reduction for higher intakes of EPA+DHA and risk of any CHD event. However, not all meta-analyses have shown benefits of $\omega 3$ fatty acids on cardiovascular outcomes [16-18]. Rizos et al. [18] reported $\omega 3$ fatty acids did not associate with a lower risk of all-cause mortality, cardiac death, sudden death, myocardial infarction or stroke, in a metaanalysis of 20 studies and 68,680 patients. The observed effect was not associated with study specific or population-specific characteristics. It is noteworthy that the relative risks for cardiac death and sudden death were reduced by $9 \%(\mathrm{P}=0.01)$ and $13 \%(\mathrm{P}=0.06)$ respectively by $\omega 3$ fatty acids, but the authors adopted a conservative statistical approach and inexplicably set the p-value for significance at 0.0063 .

Population studies have shown that increased dietary $\omega 3$ fatty acids, particularly as fatty fish, associates with a lower risk of heart failure and stroke. A meta-analysis by Djousse et al [19] comprising 176,441 subjects and 5,480 incident cases of heart failure, showed a $15 \%$ reduced relative risk $(\mathrm{p}=0.04)$ for heart failure when comparing the highest to lowest category of fish intake. Further, Mozaffarian et al [20] showed eating tuna or other broiled or baked fish, but not fried fish associated with a reduced incidence of heart failure. An analysis by Xun et al [21] that included 402,127 individuals, reported a 9\% reduced risk of total stroke in those who ate 2-4 fish meals/week relative to those that ate less than one fish meal per month. The protective effect was only evident with ischaemic stroke. 


\section{Evidence from Randomised Controlled Trials}

Several large randomised controlled trials have confirmed $\omega 3$ fatty acids benefit patients with CHD. The Diet and Reinfarction Trial (DART) in 2,033 men with recent myocardial infarction showed $\omega 3$ fatty acids as oily fish or fish oil capsules reduced all-cause mortality by $29 \%$ [22]. The Gruppo Italiano per lo Studio della Sopravvivenza nell'Infarto (GISSI) study [23] randomised 11,323 post-myocardial infarction patients and showed that after 1 year $\omega 3$ fatty acids reduced total and cardiovascular mortality, and sudden cardiac death, by $21 \%, 30 \%$ and $45 \%$, respectively. A follow-up study in nearly 7,000 patients with class II to IV heart failure (GISSI-HF), showed that $\omega 3$ fatty acids reduced total mortality by $9 \%$ and total mortality or hospitalisation for cardiovascular diseases by $8 \%$ [24]. The Japan EPA Lipid Intervention Study (JELIS) in 18,645 statin-treated hypercholesterolaemic patients, showed a $19 \%$ reduction in major cardiovascular events after 5 years in patients randomised to EPA $[25]$

It is noteworthy that several recent studies have not shown benefits of $\omega 3$ fatty acids in at-risk patient populations. In the OPERA study that included 1,516 patients undergoing cardiac surgery, $\omega 3$ fatty acids did not reduce the risk of post-operative atrial fibrillation relative to placebo [26]. The ORIGIN trial [27] in 12,536 patients with or at high risk for diabetes, reported that $\omega 3$ fatty acids did not reduce the rate of cardiovascular events. In 12,513 patients with multiple cardiovascular risk factors or atherosclerotic vascular disease but not myocardial infarction, $\omega 3$ fatty acids did not reduce cardiovascular mortality and morbidity [28]. The reasons for these negative results may relate to a number of factors. Firstly, $\omega 3$ fatty acids likely confer greater benefit in patients that have experienced a recent myocardial infarction or heart failure due to their antiarrhythmic effects. Secondly, some of the studies have had limited power to detect a reduction in sudden deaths from cardiac causes or arrhythmic events. Other important factors include trials that have used doses of $\omega 3$ fatty 
acids lower than what has been previously shown to have an effect, the presence of multiple confounding comorbidities, effects of concomitant medications and the likelihood that patients are already receiving a high level of clinical care. Studies may also have been confounded by participants already consuming a relatively high intake of $\omega 3$ fatty acids which would limit the likelihood of detecting additional benefits of $\omega 3$ fatty acid supplementation. In this regard, von Schacky [29] has suggested that most studies have recruited trial participants irrespective of their baseline blood EPA+DHA status which is an important predictor of events. Blood levels of EPA+DHA show a large inter-individual variability in responding to increased intake, leading to a significant overlap of EPA+DHA levels between intervention and control groups. Thus it has been suggested future studies should recruit participants with low baseline blood EPA+DHA levels and treat with individually tailored doses of EPA+DHA to a pre-specified target range. Similarly, Visioli [30] has suggested a lipidomic approach to screen suitable patients and correlation of the blood fatty acid profile with outcomes. An additional potential confounding factor to explain the negative findings from recent studies supplementing EPA+DHA could be a reduction in bioavailability as a result of taking supplements with other foods.

\section{Influence of $\omega 3$ Fatty Acids on Cardiovascular Risk Factors}

The benefits of EPA and DHA relate to their capacity to alter numerous physiological pathways [31] and cardiovascular risk factors including blood pressure [32-36], cardiac function [31, 34, 37], arterial compliance [38], vascular reactivity [39, 40] and lipids [41, 42]. EPA and DHA also have anti-platelet [43], anti-inflammatory [44], pro-resolving [45] and anti-oxidative actions [46]. EPA and DHA have differential effects on blood pressure, heart rate, lipids and vascular reactivity [47]. 


\subsection{Blood Pressure}

Meta-analyses of randomised controlled trials providing fish meals or fish oil supplements unequivocally show $\omega 3$ fatty acids lower blood pressure [32, 33, 35, 36]. Morris et al [35] showed blood pressure was reduced $-3.0 /-1.5 \mathrm{mmHg}$ (systolic/diastolic blood pressure) with an average dose of $4.8 \mathrm{~g} / \mathrm{day} \omega 3$ fatty acids, with the strongest effect in treated and untreated hypertensive individuals (-3.4/-2.0 $\mathrm{mmHg})$. Appel et al [32] showed blood pressure fell -5.5/3.5 $\mathrm{mmHg}$ in untreated hypertensive individuals and $-1.0 /-0.5 \mathrm{mmHg}$ in normotensives with $3 \mathrm{~g} /$ day of $\omega 3$ fatty acids. Geleijnse et al [33] reported $\omega 3$ fatty acids reduced blood pressure by $-2.1 /-1.6 \mathrm{mmHg}$, with the greatest effects in individuals older than 45 years and those that were hypertensive (-4.0/-2.5 $\mathrm{mmHg})$. Miller et al [36] showed systolic blood pressure (-1.52 $\mathrm{mm} \mathrm{Hg})$ and diastolic blood pressure $(-0.99 \mathrm{~mm} \mathrm{Hg})$ were reduced by $\omega 3$ fatty acids, with strongest effects among untreated hypertensive subjects $(-4.51 /-3.05 \mathrm{~mm} \mathrm{Hg})$. To put these blood pressure changes into perspective, a $2 \mathrm{mmHg}$ reduction in systolic blood pressure in adults is associated with a $4 \%$ fall in coronary death and $6 \%$ reduction in stroke [48].

The blood pressure-lowering effects of $\omega 3$ fatty acids are potentiated by sodium restriction [49] and antihypertensive medication.[50]. Bao et al [51] also showed that $\omega 3$ fatty acids were additive to the blood pressure-lowering effects of weight reduction in overweight treated hypertensives. Blood pressure monitored over a 24-hour period fell -6.0/-3.0 mmHg in patients consuming a daily fish meal, $-5.5 /-2.2$ in those that completed a weight loss program that reduced weight by $5.6 \mathrm{~kg}$ over 12 weeks, and -13.0/-9.3 in individuals that combined the two regimens. Mori et al [52] have also shown that the blood pressure lowering effects of $\omega 3$ fatty acids are predominantly due to DHA.

\subsection{Vascular Function and Arterial Compliance}

Human studies and experimental animal models provide convincing evidence that the blood pressure-lowering effects of $\omega 3$ fatty acids are in part due to improvements in vascular 
function [34, 39]. Further, Mori et al [40] provided evidence for differential effects of EPA and DHA on vascular function in humans. The blood pressure changes following supplementation with DHA but not EPA in dyslipidaemic patients [52], were associated with significant improvements in endothelial and smooth muscle function as well as reduced vasoconstrictor responses in the forearm microcirculation [40]. The effects of $\omega 3$ fatty acids on vasoreactivity are likely due to their incorporation into endothelial membranes with a consequent increase in membrane fluidity, calcium influx, and endogenous synthesis and release of nitric oxide. These changes likely affect enzyme activity, receptor affinity and transport capacity of the cell, including synthesis and/or release of nitric oxide. Animal studies suggest the blood pressure-lowering effects of $\omega 3$ fatty acids may also relate to alterations in catecholamines and ATP [53].

The effects of $\omega 3$ fatty acids on blood pressure are also mediated by changes in arterial compliance, which in turn is affected by endothelial function. Pase et al [38] in a metaanalysis, showed that $\omega 3$ fatty acids significantly improved both pulse wave velocity and arterial compliance.

\subsection{Cardiac Function}

Increased heart rate is a risk factor for cardiovascular death, particularly sudden death [54]. Mozaffarian et al [37] showed in a meta-analysis that $\omega 3$ fatty acids reduce heart rate by -1.6 bpm, with a greater reduction in individuals with a baseline heart rate greater than $69 \mathrm{bpm}$ $(-2.5 \mathrm{bpm})$ and in those studies of longer than 12 weeks duration $(-2.5 \mathrm{bpm})$. These data suggest the antihypertensive effects of $\omega 3$ fatty acids associate with a significant cardiac component possibly mediated by effects on cardiac myocytes, autonomic nerve function or $\beta$ adrenoreceptor activity. Using continuous 24-hour measurements of heart rate, Bao et al [51] showed that a combination of weight loss and dietary $\omega 3$ fatty acids significantly reduced heart rate in overweight treated hypertensive patients. Heart rate was reduced $4.3 \mathrm{bpm}$ by a 
daily fish meal, $1.8 \mathrm{bpm}$ following weight loss and $6.1 \mathrm{bpm}$ by a combination of the two regimens. Mori et al [52] also showed DHA had a greater effect than EPA in reducing heart rate in humans [52]. The mechanisms by which $\omega 3$ fatty acids affect heart rate likely relate to their incorporation into myocardial cells and altering electrophysiological function [55].

Studies in humans show that $\omega 3$ fatty acids increase heart rate variability in patients at high risk of sudden cardiac death and in healthy individuals. In this regard, Xin et al [56] in a meta-analysis showed that short term $\omega 3$ fatty acids favourably affect the frequency domain of heart rate variability as indicated by enhancement of vagal tone. Heart rate variability is a surrogate index of autonomic nerve function and low heart rate variability independently predicts cardiovascular disease mortality in patients with coronary artery disease or chronic heart failure [57] and in healthy populations [58]. These data suggest enhanced vagal tone may be an important mechanism underlying the antiarrhythmic effect of $\omega 3$ fatty acids.

\subsection{Plasma Lipids}

$\omega 3$ Fatty acids have very little effect on total cholesterol and LDL-C [41, 42], but they increase HDL-C [59, 60]. The greatest effect of $\omega 3$ fatty acids is on triglycerides with reductions of $20-30 \%$ reported [31]. Mori et al [60,61] further showed the triglyceridelowering actions of $\omega 3$ fatty acids were attributable to both EPA and DHA. The fall in plasma triglycerides with $\omega 3$ fatty acids is due to a reduction in hepatic VLDL-cholesterol synthesis. The mechanisms include reduced fatty acid availability for triglyceride synthesis as a result of decreased de novo lipogenesis, a reduction in the delivery of non-esterified fatty acids to the liver, increased fatty acid $\beta$-oxidation, altered enzymatic activity for triglyceride assembly in the liver and increased hepatic synthesis of phospholipids instead of triglycerides [41, 42, 62]. Clinical trials show $\omega 3$ fatty acids can compliment lipid lowering therapy in dyslipidaemic patients. In this regard, Chan et al [63] showed that $\omega 3$ fatty acids in addition to statin therapy resulted in optimal changes in the lipid profile as reflected by decreased plasma triglycerides 
and increased HDL-C. High-doses of $\omega 3$ fatty acids can also be useful adjunct therapy in the treatment of hypertriglyceridaemia.

\subsection{Glucose Homeostasis and Diabetes}

Meta-analyses have consistently shown that $\omega 3$ fatty acids have no significant effect on fasting glucose, insulin sensitivity or glycated haemoglobin in patients with Type 2 diabetes [64]. A systematic review of 540,184 individuals and 25,670 cases of incident diabetes mellitus showed fish and/or seafood consumption, or consumption of EPA+DHA, were not significantly associated with risk of diabetes [65].

\subsection{Platelet Function and Thrombosis}

The inhibitory effects of $\omega 3$ fatty acids on thrombosis are related to their capacity to reduce the risk for sudden cardiac death and all-cause mortality [8, 22, 23]. Knapp et al [66] showed that $\omega 3$ fatty acids decreased thromboxane $\mathrm{A}_{2}\left(\mathrm{TXA}_{2}\right)$ a vasoconstrictor and aggregator derived from arachidonic acid, and increased thromboxane $\mathrm{A}_{3}\left(\mathrm{TXA}_{3}\right)$ the analogous but substantially less biologically active EPA-derived metabolite (Figure 2). Fischer et al [67]<smiles>CCCCC[C@H](O)/C=C/[C@H]1O[C@H]2CC[C@@H]1O2</smiles>

Thromboxane $\mathrm{A}_{2}$

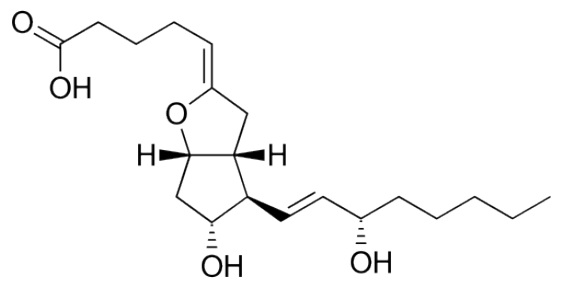

Prostaglandin $\mathrm{I}_{2}$<smiles>CC/C=C\C[C@H](O)/C=C/C1OC2CC[C@H]1O2</smiles>

Thromboxane $\mathrm{A}_{3}$<smiles>CC/C=C\C[C@H](O)/C=C/[C@H]1[C@H](O)C[C@@H]2O/C(=C\CCCC(=O)O)C[C@@H]21</smiles>

Prostaglandin $\mathrm{I}_{3}$

FIGURE 2: Structure of thromboxane $\mathrm{A}_{2}\left(\mathrm{TXA}_{2}\right)$ and prostaglandin $\mathrm{I}_{2}\left(\mathrm{PGI}_{2}\right)$ from 
arachidonic acid, and thromboxane $\mathrm{A}_{3}\left(\mathrm{TXA}_{3}\right)$ and prostaglandin $\mathrm{I}_{3}\left(\mathrm{PGI}_{3}\right)$ from EPA. showed $\omega 3$ fatty acids increased prostaglandin $\mathrm{I}_{3}\left(\mathrm{PGI}_{3}\right)$ derived from EPA, without a fall in prostaglandin $\mathrm{I}_{2}\left(\mathrm{PGI}_{2}\right)$ which derives from arachidonic acid (Figure 2). $\mathrm{PGI}_{3}$ is equipotent in its vasodilatory and anti-aggregatory activities to $\mathrm{PGI}_{2}$. An hypothesis suggests that an overall increase in total $\mathrm{PGI}_{2}$ and $\mathrm{PGI}_{3}$ formation in conjunction with reduced total thromboxane $\left(\mathrm{TXA}_{2}\right.$ and $\mathrm{TXA}_{3}$ ), could favourably alter thrombotic, endothelial and vascular responses following dietary $\omega 3$ fatty acids. Other factors that could contribute to a decrease in clinical atherothrombosis include improvements in blood rheology [68] and reduced PDGF-like proteins [69] following $\omega 3$ fatty acids. Mori et al [70] have also shown that the antiaggregatory effects of $\omega 3$ fatty acids are influenced by the background level of dietary fat and the source of $\omega 3$ fatty acids.

\subsection{Inflammation}

The anti-inflammatory and immunomodulatory effects of $\omega 3$ fatty acids are mediated via attenuation of inflammatory eicosanoids and leukotrienes, cytokines, oxidative stress, and by altering endothelial and cell-cell activation, and immune cell function [44]. EPA is a preferred substrate of the lipoxygenase pathway leading to the formation of the relatively inactive leukotriene $\mathrm{B}_{5}\left(\mathrm{LTB}_{5}\right)$ at the expense of leukotriene $\mathrm{B}_{4}\left(\mathrm{LTB}_{4}\right)$; the latter derived from arachidonic acid is a potent chemotactic factor for leukocytes [44].<smiles>CCCCC/C=C\C[C@H](O)/C=C/C=C/C=C/[C@H](O)CCCC(=O)O</smiles>

LTB $_{4}$<smiles>CC/C=C\C/C=C\CC(O)/C=C/C=C/C=C/[C@H](O)CCCC(=O)O</smiles>

LTB $_{5}$

FIGURE 3: Structure of leukotriene $\mathrm{B}_{4}\left(\mathrm{LTB}_{4}\right)$ and leukotriene $\mathrm{B}_{5}\left(\mathrm{LTB}_{5}\right)$ from arachidonic 
acid and EPA, respectively.

$\omega 3$ Fatty acids reduce ex vivo formation of pro-inflammatory cytokines following stimulation of monocytes/lymphocytes with lipopolysaccharide [44]. In-vitro. $\omega 3$ fatty acids have been shown to decrease the expression of pro-inflammatory cytokines, cell-adhesion molecules and monocyte adhesion to endothelial cells [71]. Attenuation in adhesion molecule expression by $\omega 3$ fatty acids was accompanied by decreased binding of human lymphocytes and monocytes to cytokine-stimulated endothelial cells [71].

\subsection{Lipid mediators of inflammation resolution}

Evidence shows the resolution of inflammation is an active process accompanied by the biosynthesis of potent lipid mediators that stimulate the resolution of inflammation with consequent return to tissue homeostasis. Serhan et al [45] have described chemically and functionally distinct mediators, including E-series resolvins derived from EPA via P450 metabolism or aspirin-acetylated cyclooxygenase (COX-2), and D-series resolvins, protectins and maresins derived from DHA via lipoxygenase or aspirin acetylated COX-2 (Figure 4)

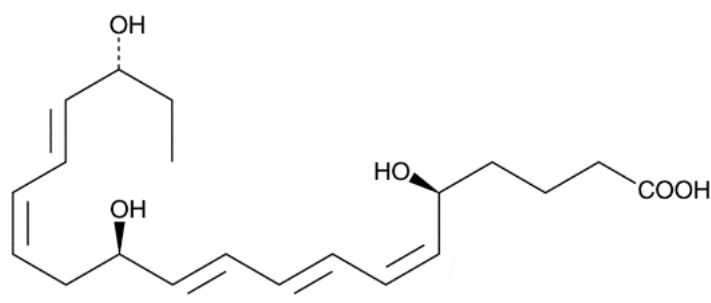

RvE1

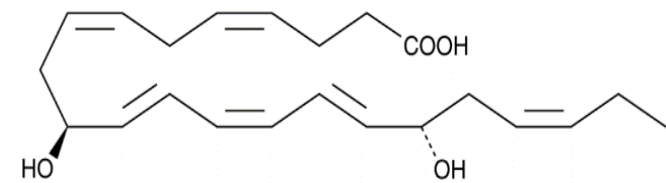

10(S),17(S)-DiHDHA (Protectin $D_{1}$ )

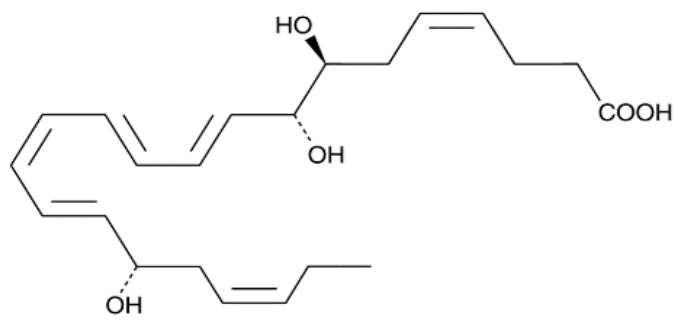

RvD1

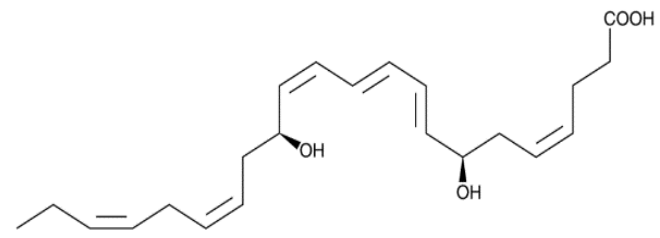

7(R)-Maresin1

FIGURE 4: Structure of resolvin E1 (RvE1) from EPA, and resolvin D1 (RvD1), protectin 
D1 and 7(R)-Maresin1 from EPA.

[45]. These mediators act via G-coupled protein receptors and have potent anti-inflammatory and pro-resolving actions that increase with time during the inflammatory process [45]. Lipid mediators of inflammation resolution reduce airway inflammation, colitis, arthritis and postoperative pain [45]. Studies in humans are providing convincing evidence that dietary $\omega 3$ fatty acids increase blood and tissue levels of lipid mediators of inflammation resolution [72]. Mori et al have shown that $\omega 3$ fatty acid supplementation increased plasma lipid mediators of inflammation resolution in healthy adults $[73,74]$, in individuals with the metabolic syndrome [75], arthritis [76], chronic kidney disease [77] and in placental tissue of pregnant women [78].

\subsection{Oxidative Stress}

Increasing evidence shows abnormal production of free radicals leads to increased stress on cellular structures and causes changes in molecular pathways that underpins the pathogenesis of a number of human diseases, including cardiovascular and neurological diseases, and cancer. In this regard, Mori et al [46] have shown plasma and urinary $\mathrm{F}_{2^{-}}$ isoprostanes, measured by gas chromatography-mass spectrometery, are reduced following $\omega 3$ fatty acids. $F_{2}$-isoprostanes are lipid peroxidation products derived from the nonenzymatic free radical oxidation of arachidonic acid in membrane lipids and are considered the most reliable biomarkers of in vivo lipid peroxidative damage. The mechanisms by which $\omega 3$ fatty acids attenuate oxidative stress likely involve decreased leukocyte activation and immunomodulatory actions [79].

\subsection{Effects on Plaque Stabilization}

There is some evidence that $\omega 3$ fatty acids may alter plaque morphology, suggestive of increased stability. These findings could represent an important mechanism by which $\omega 3$ fatty acids reduce ischaemic cardiovascular events. In this regard, Thies et al [80] showed that $\omega 3$ 
fatty acids were readily incorporated into the atherosclerotic plaque of patients with carotid atherosclerotic disease undergoing carotid endarterectomy and that this associated with a reduced number of macrophages in the plaque.

\subsection{The $\omega 3$ Index}

Harris and von Schacky [81] have proposed a measure, termed the Omega-3 Index and defined as the sum of EPA+DHA in erythrocytes, that may serve as a marker for cardiovascular health and CHD. They further suggested that an Omega-3 Index of $\geq 8 \%$ associates with cardioprotective benefits whereas an index of $\leq 4 \%$ gives the least cardioprotection [81], an hypothesis supported by data from studies in patients with acute coronary syndrome [82] and stable CHD [83].

\section{Guidelines for $\omega 3$ Fatty Acid Intake}

The American Heart Association [84] and other global health authorities have made population-based dietary recommendations for $\omega 3$ fatty acids. Current guidelines suggest individuals should consume approximately $500 \mathrm{mg}$ /day of EPA and DHA [85], which is achievable with at least two $100 \mathrm{~g}$ serves of fish per week, preferably oil fish species such as fresh tuna, salmon, mackerel, herring and sardines [86], and preferably broiled or baked, but not fried [20]. The most practical recommendation for increasing dietary $\omega 3$ fatty acids is to incorporate fish as part of a healthy diet that includes increased fruits and vegetables, and moderation of salt intake. Individuals with CHD should be encouraged to increase $\omega 3$ fatty acid to approximately $1 \mathrm{~g}$ daily [86], based largely on data from clinical trials [23]. $\omega 3$ Fatty acid supplements at doses $2-4 \mathrm{~g} /$ day should be considered in combination with other lipid therapies in patients with hypertriglyceridaemia [86]. These doses of $\omega 3$ fatty acids are achievable with high-quality encapsulated $\omega 3$ fatty acid supplements free of contaminants.

$\omega 3$ Fatty acid supplements do not associate with adverse effects other than occasional 
gastrointestinal upset and a "fishy burp". The latter is preventable by taking capsules with a cold beverage and a meal. Although there are some concerns that high intakes of $\omega 3$ fatty acids could potentially increase the risk of bleeding when taken in conjunction with antiplatelet or anticoagulant medications, there was little evidence to date of adverse effects. However, Bays [87] suggested clinicians should be mindful of increased bleeding as a theoretical possibility. Further, some clinicians advise patients to discontinue fish oil supplements 4-7 days prior to invasive procedures with the highest risk for bleeding complications. Postoperatively, clinicians should consider the cardiovascular advantages of recommencing $\omega 3$ fatty acid supplements given cardiovascular and thrombotic events are often common complications after major surgeries.

It should be noted that some fish species may contain levels of methylmercury and environmental contaminants, which is very much dependent on the location where these fish are caught. However, these substances are only present at highest levels in predatory fish such as shark, swordfish, marlin and larger species of tuna, and marine mammals. Health advisory bodies suggest women who are either pregnant or may become pregnant, lactating women and young children, to limit consumption of such fish species in order to minimize their exposure to these contaminants [86].

\section{Conclusions}

Current evidence strongly supports the concept that $\omega 3$ fatty acids beneficially influence a number of cardiometabolic risk factors, with the strongest evidence from studies examining dietary fish consumption. Dietary intake of fish has consistently been associated with lower incident rates of heart failure, lower sudden cardiac death, stroke and myocardial infarction. The recent American Heart Association Science Advisory [84] concluded that treatment with $\omega 3$ fatty acids is reasonable for (1) secondary prevention of CHD and sudden cardiac death 
among patients with prevalent CHD; and (2) secondary prevention of adverse outcomes in patients with heart failure. The Australian National Heart Foundation supports these recommendations and suggests evidence continues to be positive for the role of $\omega 3$ fatty acids in the treatment of hypertriglyceridaemia [88]. Whilst there may be some benefit in atrial fibrillation and hypertension, further trials are required to support any firm recommendations [88]. $\omega 3$ Fatty acids do not have adverse interactions with lipid-lowering drugs or antihypertensive and antithrombotic medications. Small doses of $1 \mathrm{~g} /$ day are achievable with consumption of 2-3 oily fish meals/week. Higher doses up to at least 4g/day have no clinically significant adverse effects and are achievable with encapsulated preparations now available. Overall there is good evidence that increased dietary $\omega 3$ fatty acids should be encouraged. In particular, fish should be considered an important component of a healthy diet.

\section{Acknowledgements}

This review is dedicated in the memory of the late Professor Emilio Ghisalberti who was responsible for stimulating the author's research career and remained a close friend. Gratitude is extended to my colleagues and past students that have made most the research findings from my laboratory possible. Aspects of the research from my laboratory presented in this review was supported by grants from the National Health \& Medical Research Council of Australia; the National Heart Foundation of Australia; the Fish Oil Test Materials Program of the National Institutes of Health/Department of Commerce, USA; and the Medical Research Foundation of Royal Perth Hospital, Perth, Western Australia. The author is supported in part by a Research Fellowship from the National Health and Medical Research Council (NHMRC) of Australia (1042255).

\section{Conflicts Of Interest: none}




\section{References}

[1] H.O. Bang, J. Dyerberg, A.B. Nielson, Plasma Lipid and Lipoprotein Pattern in Greenlandic West-Coast Eskimos, Lancet 1(7710) (1971) 1143-1146.

[2] H.O. Bang, J. Dyerberg, N. Hjorne, Composition of Food Consumed by Greenland Eskimos, Acta Med Scand 200(1-2) (1976) 69-73.

[3] H.O. Bang, J. Dyerberg, H.M. Sinclair, The Composition of the Eskimo Food in Northwestern Greenland, Am J Clin Nutr 33(12) (1980) 2657-2661.

[4] M. Wada, C.J. DeLong, Y.H. Hong, C.J. Rieke, I. Song, R.S. Sidhu, C. Yuan, M. Warnock, A.H. Schmaier, C. Yokoyama, E.M. Smyth, S.J. Wilson, G.A. FitzGerald, R.M. Garavito, D.X. Sui, J.W. Regan, W.L. Smith, Enzymes and receptors of prostaglandin pathways with arachidonic acid-derived versus eicosapentaenoic acidderived substrates and products, J Biol Chem 282(31) (2007) 22254-66.

[5] S.P. Tull, C.M. Yates, B.H. Maskrey, V.B. O'Donnell, J. Madden, R.F. Grimble, P.C. Calder, G.B. Nash, G.E. Rainger, Omega-3 Fatty acids and inflammation: novel interactions reveal a new step in neutrophil recruitment, Plos Biol 7(8) (2009) e1000177.

[6] J.T. Brenna, N. Salem, A.J. Sinclair, S.C. Cunnane, Issfal, alpha-Linolenic acid supplementation and conversion to n-3 long-chain polyunsaturated fatty acids in humans, Prost Leuk Essent Fatty Acids 80(2-3) (2009) 85-91.

[7] H.C. Bucher, P. Hengstler, C. Schindler, G. Meier, N-3 polyunsaturated fatty acids in coronary heart disease: a meta-analysis of randomized controlled trials, Am J Med 112(4) (2002) 298-304.

[8] K. He, Y.Q. Song, M.L. Daviglus, K. Liu, L. Van Horn, A.R. Dyer, P. Greenland, Accumulated evidence on fish consumption and coronary heart disease mortality - A meta-analysis of cohort studies, Circulation 109(22) (2004) 2705-2711.

[9] C.C. Wang, W.S. Harris, M. Chung, A.H. Lichtenstein, E.M. Balk, B. Kupelnick, H.S. Jordan, J. Lau, n-3 fatty acids from fish or fish-oil supplements, but not alpha-linolenic acid, benefit cardiovascular disease outcomes in primary- and secondary-prevention studies: a systematic review, Am J Clin Nutr 84(1) (2006) 5-17.

[10] S.P. Whelton, J. He, P.K. Whelton, P. Muntner, Meta-analysis of observational studies on fish intake and coronary heart disease, Am J Cardiol 93(9) (2004) 1119-23.

[11] P.E. Marik, J. Varon, Omega-3 Dietary Supplements and the Risk of Cardiovascular Events: A Systematic Review, Clin Cardiol 32(7) (2009) 365-372.

[12] K. Musa-Veloso, M.A. Binns, A. Kocenas, C. Chung, H. Rice, H. Oppedal-Olsen, H. Lloyd, S. Lemke, Impact of low v. moderate intakes of long-chain n-3 fatty acids on risk of coronary heart disease, Br J Nutr 106(8) (2011) 1129-1141.

[13] Y.T. Zhao, Q. Chen, Y.X. Sun, X.B. Li, P. Zhang, Y. Xu, J.H. Guo, Prevention of sudden cardiac death with omega-3 fatty acids in patients with coronary heart disease: a meta-analysis of randomized controlled trials, Annals Med 41(4) (2009) 301-10.

[14] D.D. Alexander, P.E. Miller, M.E. Van Elswyk, C.N. Kuratko, L.C. Bylsma, A MetaAnalysis of Randomized Controlled Trials and Prospective Cohort Studies of Eicosapentaenoic and Docosahexaenoic Long-Chain Omega-3 Fatty Acids and Coronary Heart Disease Risk, Mayo Clin Proc 92(1) (2017) 15-29.

[15] K.C. Maki, O.M. Palacios, M. Bell, P.P. Toth, Use of supplemental long-chain omega-3 fatty acids and risk for cardiac death: An updated meta-analysis and review of research gaps, J Clin Lipidol (2017) http://dx.doi.org/10.1016/j.jacl.2017.07.010.

[16] S. Kotwal, M. Jun, D. Sullivan, V. Perkovic, B. Neal, Omega 3 Fatty Acids and Cardiovascular Outcomes Systematic Review and Meta-Analysis, Circ-Cardiovasc Qual 5(6) (2012) 808-818. 
[17] S.M. Kwak, S.K. Myung, Y.J. Lee, H.G. Seo, Efficacy of omega-3 fatty acid supplements (eicosapentaenoic acid and docosahexaenoic acid) in the secondary prevention of cardiovascular disease: a meta-analysis of randomized, double-blind, placebo-controlled trials, Arch Intern Med 172(9) (2012) 686-94.

[18] E.C. Rizos, E.E. Ntzani, E. Bika, M.S. Kostapanos, M.S. Elisaf, Association Between Omega-3 Fatty Acid Supplementation and Risk of Major Cardiovascular Disease Events A Systematic Review and Meta-analysis, JAMA 308(10) (2012) 1024-1033.

[19] L. Djousse, A.O. Akinkuolie, J.H.Y. Wu, E.L. Ding, J.M. Gaziano, Fish consumption, omega-3 fatty acids and risk of heart failure: A meta-analysis, Clin Nutr 31(6) (2012) 846-853.

[20] D. Mozaffarian, C.L. Bryson, R.N. Lemaitre, G.L. Burke, D.S. Siscovick, Fish intake and risk of incident heart failure, J Am Coll Cardiol 45(12) (2005) 2015-21.

[21] P. Xun, B. Qin, Y. Song, Y. Nakamura, T. Kurth, S. Yaemsiri, L. Djousse, K. He, Fish consumption and risk of stroke and its subtypes: accumulative evidence from a metaanalysis of prospective cohort studies, Eur J Clin Nutr 66(11) (2012) 1199-207.

[22] M.L. Burr, J.F. Gilbert, R.M. Holliday, P.C. Elwood, A.M. Fehily, S. Rogers, P.M. Sweetnam, N.M. Deadman, Effects of Changes in Fat, Fish, and Fiber Intakes on Death and Myocardial Reinfarction - Diet and Reinfarction Trial (Dart), Lancet 2(8666) (1989) 757-761.

[23] F. Valagussa, M.G. Franzosi, E. Geraci, N. Mininni, G.L. Nicolosi, M. Santini, L. Tavazzi, C. Vecchio, R. Marchioli, E. Bomba, C. Chieffo, A.P. Maggioni, C. Schweiger, G. Tognoni, F. Barzi, A.V. Flamminio, R.M. Marfisi, M. Olivieri, C. Pera, A. Polidoro, E. Santoro, R. Zama, L. Pagliaro, E. Correale, A. Del Favero, U. Loi, E. Marubini, L. Campolo, A. Casari, G. Di Minno, M.B. Donati, M. Galli, M. Gattone, S. Garattini, M. Mancini, P. Marino, G.M. Santoro, C. Scardulla, G. Specchia, A. Cericola, D. Di Gregorio, R. Di Mascio, G. Levantesi, L. Mantini, G. Mastrogiuseppe, C. Tucci, P. Mocarelli, R. Baldinelli, F. Ceriotti, A. Colonna, C. Cortese, G. Fortunato, C. Franzini, F. Gonano, M.S. Graziani, G.-P. Investigators, Dietary supplementation with n-3 polyunsaturated fatty acids and vitamin $\mathrm{E}$ after myocardial infarction: results of the GISSI-Prevenzione trial, Lancet 354(9177) (1999) 447-455.

[24] L. Tavazzi, A.P. Maggioni, R. Marchioli, S. Barlera, M.G. Franzosi, R. Latini, D. Lucci, G.L. Nicolosi, M. Porcu, G. Tognoni, G.H. Investigators, Effect of n-3 polyunsaturated fatty acids in patients with chronic heart failure (the GISSI-HF trial): a randomised, double-blind, placebo-controlled trial, Lancet 372(9645) (2008) 1223-1230.

[25] M. Yokoyama, H. Origasa, M. Matsuzaki, Y. Matsuzawa, Y. Saito, Y. Ishikawa, S. Oikawa, J. Sasaki, H. Hishida, H. Itakura, T. Kita, A. Kitabatake, N. Nakaya, T. Sakata, K. Shimada, K. Shirato, J. Investigators, Effects of eicosapentaenoic acid on major coronary events in hypercholesterolaemic patients (JELIS): a randomised openlabel, blinded endpoint analysis, Lancet 369(9567) (2007) 1090-1098.

[26] D. Mozaffarian, R. Marchioli, A. Macchia, M.G. Silletta, P. Ferrazzi, T.J. Gardner, R. Latini, P. Libby, F. Lombardi, P.T. O'Gara, R.L. Page, L. Tavazzi, G. Tognoni, O. Investigators, Fish Oil and Postoperative Atrial Fibrillation The Omega-3 Fatty Acids for Prevention of Post-operative Atrial Fibrillation (OPERA) Randomized Trial, JAMA 308(19) (2012) 2001-2011.

[27] J. Bosch, H.C. Gerstein, G.R. Dagenais, R. Diaz, L. Dyal, H. Jung, A.P. Maggiono, J. Probstfield, A. Ramachandran, M.C. Riddle, L.E. Ryden, S. Yusuf, n-3 Fatty Acids and Cardiovascular Outcomes in Patients with Dysglycemia, New Engl J Med 367(4) (2012) 309-318.

[28] M.C. Roncaglioni, M. Tombesi, F. Avanzini, S. Barlera, V. Caimi, P. Longoni, I. Marzona, V. Milani, M.G. Silletta, G. Tognoni, R. Marchioli, R.P.S. Collaborativ, n-3 
Fatty Acids in Patients with Multiple Cardiovascular Risk Factors, New Engl J Med 368(19) (2013) 1800-1808.

[29] C. von Schacky, Omega-3 fatty Acids in cardiovascular disease - An uphill battle, Prost Leuk Essent Fatty Acids 92 (2015) 41-47.

[30] F. Visioli, Lipidomics to Assess Omega 3 Bioactivity, J Clin Med 4(9) (2015) 1753-60.

[31] D. Mozaffarian, J.H. Wu, Omega-3 fatty acids and cardiovascular disease: effects on risk factors, molecular pathways, and clinical events, J Am Coll Cardiol 58(20) (2011) 2047-67.

[32] L.J. Appel, E.R. Miller, A.J. Seidler, P.K. Whelton, Does Supplementation of Diet with Fish-Oil Reduce Blood-Pressure - a Metaanalysis of Controlled Clinical-Trials, Arch Int Med 153(12) (1993) 1429-1438.

[33] J.M. Geleijnse, E.J. Giltay, D.E. Grobbee, A.R.T. Donders, F.J. Kok, Blood pressure response to fish oil supplementation: metaregression analysis of randomized trials, $\mathrm{J}$ Hypertens 20(8) (2002) 1493-1499.

[34] T.A. Mori, V. Burke, L.J. Beilin, Dietary Fats and Blood Pressure, Elsevier, Philadelphia, 2007.

[35] M.C. Morris, F. Sacks, B. Rosner, Does Fish-Oil Lower Blood-Pressure - a Metaanalysis of Controlled Trials, Circulation 88(2) (1993) 523-533.

[36] P.E. Miller, M. Van Elswyk, D.D. Alexander, Long-chain omega-3 fatty acids eicosapentaenoic acid and docosahexaenoic acid and blood pressure: a meta-analysis of randomized controlled trials, Am J Hypertens 27(7) (2014) 885-96.

[37] D. Mozaffarian, A. Geelen, I.A. Brouwer, J.M. Geleijnse, P.L. Zock, M.B. Katan, Effect of fish oil on heart rate in humans - A meta-analysis of randomized controlled trials, Circulation 112(13) (2005) 1945-1952.

[38] M.P. Pase, N.A. Grima, J. Sarris, Do long-chain n-3 fatty acids reduce arterial stiffness? A meta-analysis of randomised controlled trials, Br J Nutr 106(7) (2011) 974-980.

[39] J.P. Chin, Marine oils and cardiovascular reactivity, Prost Leuk Essent Fatty Acids 50(5) (1994) 211-22.

[40] T.A. Mori, G.F. Watts, V. Burke, E. Hilme, I.B. Puddey, L.J. Beilin, Differential effects of eicosapentaenoic acid and docosahexaenoic acid on vascular reactivity of the forearm microcirculation in hyperlipidemic, overweight men, Circulation 102(11) (2000) 12649.

[41] W.S. Harris, n-3 fatty acids and lipoproteins: comparison of results from human and animal studies, Lipids 31(3) (1996) 243-52.

[42] W.S. Harris, n-3 fatty acids and serum lipoproteins: human studies, Am J Clin Nutr 65(5 Suppl) (1997) 1645S-1654S.

[43] H.R. Knapp, Dietary fatty acids in human thrombosis and hemostasis, Am J Clin Nutr 65(5) (1997) S1687-S1698.

[44] P.C. Calder, Omega-3 polyunsaturated fatty acids and inflammatory processes: nutrition or pharmacology?, Br J Clin Pharmaco 75(3) (2013) 645-662.

[45] C.N. Serhan, Treating inflammation and infection in the 21st century: new hints from decoding resolution mediators and mechanisms, FASEB J 31(4) (2017) 1273-1288.

[46] E. Mas, R.J. Woodman, V. Burke, I.B. Puddey, L.J. Beilin, T. Durand, T.A. Mori, The omega-3 fatty acids EPA and DHA decrease plasma $\mathrm{F}(2)$-isoprostanes: Results from two placebo-controlled interventions, Free Rad Res 44(9) (2010) 983-90.

[47] T.A. Mori, R.J. Woodman, The independent effects of eicosapentaenoic acid and docosahexaenoic acid on cardiovascular risk factors in humans, Curr Opin Clin Nutr Metab Care 9(2) (2006) 95-104.

[48] J. Stamler, G. Rose, R. Stamler, P. Elliott, A. Dyer, M. Marmot, INTERSALT study findings. Public health and medical care implications, Hypertension 14(5) (1989) 570-7. 
[49] L. Cobiac, P.J. Nestel, L.M. Wing, P.R. Howe, A low-sodium diet supplemented with fish oil lowers blood pressure in the elderly, J Hypertens 10(1) (1992) 87-92.

[50] Y.K. Lungershausen, M. Abbey, P.J. Nestel, P.R. Howe, Reduction of blood pressure and plasma triglycerides by omega-3 fatty acids in treated hypertensives, J Hypertens 12(9) (1994) 1041-5.

[51] D.Q. Bao, T.A. Mori, V. Burke, I.B. Puddey, L.J. Beilin, Effects of dietary fish and weight reduction on ambulatory blood pressure in overweight hypertensives, Hypertension 32(4) (1998) 710-7.

[52] T.A. Mori, D.Q. Bao, V. Burke, I.B. Puddey, L.J. Beilin, Docosahexaenoic acid but not eicosapentaenoic acid lowers ambulatory blood pressure and heart rate in humans, Hypertension 34(2) (1999) 253-60.

[53] M. Hashimoto, K. Shinozuka, S. Gamoh, Y. Tanabe, M.S. Hossain, Y.M. Kwon, N. Hata, Y. Misawa, M. Kunitomo, S. Masumura, The hypotensive effect of docosahexaenoic acid is associated with the enhanced release of ATP from the caudal artery of aged rats, J Nutr 129(1) (1999) 70-76.

[54] W.B. Kannel, C. Kannel, R.S. Paffenbarger, Jr., L.A. Cupples, Heart rate and cardiovascular mortality: the Framingham Study, Am Heart J 113(6) (1987) 1489-94.

[55] A. Leaf, J.X. Kang, Y.F. Xiao, G.E. Billman, Clinical prevention of sudden cardiac death by n-3 polyunsaturated fatty acids and mechanism of prevention of arrhythmias by n-3 fish oils, Circulation 107(21) (2003) 2646-2652.

[56] W. Xin, W. Wei, X.Y. Li, Short-term effects of fish-oil supplementation on heart rate variability in humans: a meta-analysis of randomized controlled trials, Am J Clin Nutr 97(5) (2013) 926-935.

[57] J.M. Dekker, R.S. Crow, A.R. Folsom, P.J. Hannan, D. Liao, C.A. Swenne, E.G. Schouten, Low heart rate variability in a 2-minute rhythm strip predicts risk of coronary heart disease and mortality from several causes - The ARIC study, Circulation 102(11) (2000) 1239-1244.

[58] J.M. Dekker, E.G. Schouten, P. Klootwijk, J. Pool, C.A. Swenne, D. Kromhout, Heart rate variability from short electrocardiographic recordings predicts mortality from all causes in middle-aged and elderly men - The Zutphen study, Am J Epidemiol 145(10) (1997) 899-908.

[59] T.A. Mori, D.Q. Bao, V. Burke, I.B. Puddey, G.F. Watts, L.J. Beilin, Dietary fish as a major component of a weight-loss diet: effect on serum lipids, glucose, and insulin metabolism in overweight hypertensive subjects, Am J Clin Nutr 70(5) (1999) 817-25.

[60] T.A. Mori, V. Burke, I.B. Puddey, G.F. Watts, D.N. O'Neal, J.D. Best, L.J. Beilin, Purified eicosapentaenoic and docosahexaenoic acids have differential effects on serum lipids and lipoproteins, LDL particle size, glucose, and insulin in mildly hyperlipidemic men, Am J Clin Nutr 71(5) (2000) 1085-94.

[61] R.J. Woodman, T.A. Mori, V. Burke, I.B. Puddey, G.F. Watts, L.J. Beilin, Effects of purified eicosapentaenoic and docosahexaenoic acids on glycemic control, blood pressure, and serum lipids in type 2 diabetic patients with treated hypertension, Am J Clin Nutr 76(5) (2002) 1007-15.

[62] W.S. Harris, D. Bulchandani, Why do omega-3 fatty acids lower serum triglycerides?, Curr Opin Lipidol 17(4) (2006) 387-93.

[63] D.C. Chan, G.F. Watts, T.A. Mori, P.H. Barrett, L.J. Beilin, T.G. Redgrave, Factorial study of the effects of atorvastatin and fish oil on dyslipidaemia in visceral obesity, Eur J Clin Invest 32(6) (2002) 429-36.

[64] A.O. Akinkuolie, J.S. Ngwa, J.B. Meigs, L. Djousse, Omega-3 polyunsaturated fatty acid and insulin sensitivity: A meta-analysis of randomized controlled trials, Clin Nutr 30(6) (2011) 702-707. 
[65] J.H.Y. Wu, R. Micha, F. Imamura, A. Pan, M.L. Biggs, O. Ajaz, L. Djousse, F.B. Hu, D. Mozaffarian, Omega-3 fatty acids and incident type 2 diabetes: a systematic review and meta-analysis, Br J Nutr 107 (2012) S214-S227.

[66] H.R. Knapp, I.A. Reilly, P. Alessandrini, G.A. FitzGerald, In vivo indexes of platelet and vascular function during fish-oil administration in patients with atherosclerosis, $\mathrm{N}$ Engl J Med 314(15) (1986) 937-42.

[67] S. Fischer, P.C. Weber, Prostaglandin-I3 Is Formed Invivo in Man after Dietary Eicosapentaenoic Acid, Nature 307(5947) (1984) 165-168.

[68] I.J. Cartwright, A.G. Pockley, J.H. Galloway, M. Greaves, F.E. Preston, The Effects of Dietary Omega-3 Poly-Unsaturated Fatty-Acids on Erythrocyte-Membrane Phospholipids, Erythrocyte Deformability and Blood-Viscosity in Healthy-Volunteers, Atherosclerosis 55(3) (1985) 267-281.

[69] C. von Schacky, K. Baumann, P. Angerer, The effect of n-3 fatty acids on coronary atherosclerosis: Results from SCIMO, an angiographic study, background and implications, Lipids 36 (2001) S99-S102.

[70] T.A. Mori, L.J. Beilin, V. Burke, J. Morris, J. Ritchie, Interactions between dietary fat, fish, and fish oils and their effects on platelet function in men at risk of cardiovascular disease, Arterioscler Thromb Vasc Biol 17(2) (1997) 279-86.

[71] R. De Caterina, J.K. Liao, P. Libby, Fatty acid modulation of endothelial activation, Am J Clin Nutr 71(1) (2000) 213s-223s.

[72] A.E. Barden, E. Mas, T.A. Mori, n-3 Fatty acid supplementation and proresolving mediators of inflammation, Curr Opin Lipidol 27(1) (2016) 26-32.

[73] A. Barden, E. Mas, K.D. Croft, M. Phillips, T.A. Mori, Short-term n-3 fatty acid supplementation but not aspirin increases plasma proresolving mediators of inflammation, J Lipid Res 55(11) (2014) 2401-7.

[74] E. Mas, K.D. Croft, P. Zahra, A. Barden, T.A. Mori, Resolvins D1, D2, and Other Mediators of Self-Limited Resolution of Inflammation in Human Blood following n-3 Fatty Acid Supplementation, Clin Chem 58(10) (2012) 1476-1484.

[75] A.E. Barden, E. Mas, K.D. Croft, M. Phillips, T.A. Mori, Specialized proresolving lipid mediators in humans with the metabolic syndrome after n-3 fatty acids and aspirin, Am J Clin Nutr 102(6) (2015) 1357-1364.

[76] A.E. Barden, M. Moghaddami, E. Mas, M. Phillips, L.G. Cleland, T.A. Mori, Specialised pro-resolving mediators of inflammation in inflammatory arthritis, Prost Leuk Essent Fatty Acids 107 (2016) 24-29.

[77] E. Mas, A. Barden, V. Burke, L.J. Beilin, G.F. Watts, R.C. Huang, I.B. Puddey, A.B. Irish, T.A. Mori, A randomized controlled trial of the effects of n-3 fatty acids on resolvins in chronic kidney disease, Clin Nutr 35(2) (2016) 331-336.

[78] J.A. Keelan, E. Mas, N. D'Vaz, J.A. Dunstan, S. Li, A.E. Barden, P.J. Mark, B.J. Waddell, S.L. Prescott, T.A. Mori, Effects of maternal n-3 fatty acid supplementation on placental cytokines, pro-resolving lipid mediators and their precursors, Reproduction 149(2) (2015) 171-8.

[79] E. Giordano, F. Visioli, Long-chain omega 3 fatty acids: molecular bases of potential antioxidant actions, Prost Leuk Essent Fatty Acids 90(1) (2014) 1-4.

[80] F. Thies, J.M.C. Garry, P. Yaqoob, K. Rerkasem, J. Williams, C.P. Shearman, P.J. Gallagher, P.C. Calder, R.F. Grimble, Association of n-3 polyunsaturated fatty acids with stability of atherosclerotic plaques: a randomised controlled trial, Lancet 361(9356) (2003) 477-485.

[81] W.S. Harris, C. Von Schacky, The Omega-3 Index: a new risk factor for death from coronary heart disease?, Prev Med 39(1) (2004) 212-20. 
[82] R.C. Block, W.S. Harris, K.J. Reid, S.A. Sands, J.A. Spertus, EPA and DHA in blood cell membranes from acute coronary syndrome patients and controls, Atherosclerosis 197(2) (2008) 821-828.

[83] J.V. Pottala, S. Garg, B.E. Cohen, M.A. Whooley, W.S. Harris, Blood Eicosapentaenoic and Docosahexaenoic Acids Predict All-Cause Mortality in Patients With Stable Coronary Heart Disease The Heart and Soul Study, Circ-Cardiovasc Qual 3(4) (2010) 406-412.

[84] D.S. Siscovick, T.A. Barringer, A.M. Fretts, J.H. Wu, A.H. Lichtenstein, R.B. Costello, P.M. Kris-Etherton, T.A. Jacobson, M.B. Engler, H.M. Alger, L.J. Appel, D.

Mozaffarian, L. American Heart Association Nutrition Committee of the Council on, H. Cardiometabolic, E. Council on, Prevention, Y. Council on Cardiovascular Disease in the, C. Council on, N. Stroke, C. Council on Clinical, Omega-3 Polyunsaturated Fatty Acid (Fish Oil) Supplementation and the Prevention of Clinical Cardiovascular Disease: A Science Advisory From the American Heart Association, Circulation 135(15) (2017) e867-e884.

[85] S.K. Gebauer, T.L. Psota, W.S. Harris, P.M. Kris-Etherton, n-3 fatty acid dietary recommendations and food sources to achieve essentiality and cardiovascular benefits, Am J Clin Nutr 83(6) (2006) 1526s-1535s.

[86] P.M. Kris-Etherton, W.S. Harris, L.J. Appel, N. Comm, Fish consumption, fish oil, omega-3 fatty acids, and cardiovascular disease, Circulation 106(21) (2002) 2747-2757.

[87] H.E. Bays, Safety considerations with omega-3 fatty acid therapy, Am J Cardiol 99(6A) (2007) 35C-43C.

[88] P. Nestel, P. Clifton, D. Colquhoun, M. Noakes, T.A. Mori, D. Sullivan, B. Thomas, Indications for Omega-3 Long Chain Polyunsaturated Fatty Acid in the Prevention and Treatment of Cardiovascular Disease, Heart Lung Circ 24(8) (2015) 769-779. 